\title{
Development of an Automated Updated Selvester QRS Scoring System using SWT-based QRS fractionation detection and classification
}

\author{
Valentina Bono, Evangelos B. Mazomenos, Taihai Chen, James A. Rosengarten, Amit Acharyya, Member, IEEE, \\ Koushik Maharatna, Member, IEEE, John M. Morgan and Nick Curzen
}

\begin{abstract}
The Selvester score is an effective means for estimating the extent of myocardial scar in a patient from lowcost ECG recordings. Automation of such a system is deemed to help implementing low-cost high-volume screening mechanisms of scar in the primary care. This article describes, for the first time to the best of our knowledge, an automated implementation of the updated Selvester scoring system for that purpose, where fractionated QRS morphologies and patterns are identified and classified using a novel Stationary Wavelet Transform (SWT) based fractionation detection algorithm. This stage informs the two principal steps of the updated Selvester scoring scheme the confounder classification and the point awarding rules. The complete system is validated on 51 ECG records of patients detected with ischemic heart disease. Validation has been carried out using manually detected confounder classes and computation of the actual score by expert cardiologists as the ground truth. Our results show that as a stand-alone system it is able to classify different confounders with $\mathbf{9 4 . 1 \%}$ accuracy whereas it exhibits 94\% accuracy in computing the actual score. When coupled with our previously proposed automated ECG delineation algorithm, that provides the input ECG parameters, the overall system shows $90 \%$ accuracy in confounder classification and $92 \%$ accuracy in computing the actual score and thereby showing comparable performance to the stand-alone system proposed here, with the added advantage of complete automated analysis without any human intervention.
\end{abstract}

Index Terms-Stationary Wavelet Transform, Electrocardiography, Selvester QRS Score, Automated ECG Processing, Myocardial Scar

\section{INTRODUCTION}

A Heart attack, known as myocardial infarction (MI), can occur following a sudden compromise in blood supply. If blood flow is not restored promptly, healing results in scar tissue.This scar tissue demonstrates different properties to healthy heart tissue, and reduces the contractile efficiency of the heart. In addition, since the scar tissue cannot propagate

Manuscript received November 22 2012; revised March 28 2013; accepted May 6 2013; Date of publication date of current version. This work was supported by E.U. ARTEMIS Joint Undertaking under the Cyclic and personcentric Health management: Integrated appRoach for hOme, mobile and clinical eNvironments - (CHIRON) Project under Grant Agreement \# 20091-100228.

V.Bono, E.B. Mazomenos, T. Chen and K. Maharatna are with the ECS School, University of Southampton, SO17 1BJ, U.K. (e-mail: $\{$ vb2f11,ebm,tc10g09,km3\}@ecs.soton.ac.uk)

J.A. Rosengarten, J.M. Morgan and N. Curzen are with the University Hospital Southampton NHS Foundation Trust, Southampton, UK. email: james@rosengarten.co.uk,jmm@hrclinic.org,nick.curzen@suht.swest.nhs.uk

A. Acharyya is with EEE Dept., IIT Hyderabad, India. email: amit_acharyya@iith.ac.in normal electrical activity, disordered conduction around an area of scar becomes a focus for formation of dangerous heart rhythms (arrhythmias).

Imaging techniques such as nuclear perfusion scanning and contrast enhanced cardiac magnetic resonance imaging (CMR) are used in clinical practice to define the presence and magnitude of scar. Quantification of scar size helps to define risk of arrhythmia and/or death, and therefore guide preventive therapies. However, these imaging modalities are not available in every hospital, nor suitable for bedside testing. On the other hand, the electrocardiogram (ECG) is widely available and can be also used in a bedside or mobile environment. Since the QRS-complex of the ECG reflects the electrical activity, or depolarisation, through ventricles, there is clinical interest in defining features from the QRS-complex that may reflect scar. As an example, disordered conduction may manifest as various additional deflections in the QRS-complex - fractionated QRS - resulting in notching, slowing or slurring of the typical ECG trace.

The Selvester QRS score was developed to quantify left ventricular scar and is based upon 50 manually measured ECG criteria, including the presence of fractionated QRS features and various QRS width and amplitude ratios [1]. The score was initially developed from computer modelling studies of the heart's activity and validated with post mortem specimens, but was limited due to its application to an ECG of normal width QRS only [2], [3]. The score was recently (2009) updated to take account of various ECG abnormalities, or confounders, such as left bundle branch block (LBBB) or left ventricular hypertrophy (LVH) that may also be present but not necessarily related to scarring. This updated version of the Selvester score demonstrates good agreement with scar measured on CMR [4], [5]. The criteria used to define an ECG confounder, and to award points for the presence of scar, rely upon the accurate detection of the delayed QRS conduction, seen as fractionated QRS and in particular notching (reversal in direction $\geq 90^{\circ}$ and notch amplitude $\geq 0.05 \mathrm{mV}$ ), or slurring/slowing (change in gradient $\leq 90^{\circ}$ ) in the QRS trace [6]. The manual calculation of the Selvester score is a time consuming process, and lacks reproducibility due to the limits of human precision, even following appropriate training, and therefore is not currently widely used in clinical practice. An automated tool for computing the Selvester score will remove these limitations and enable its effective deployment in clinical practice. Since the Selvester score provides an estimation 


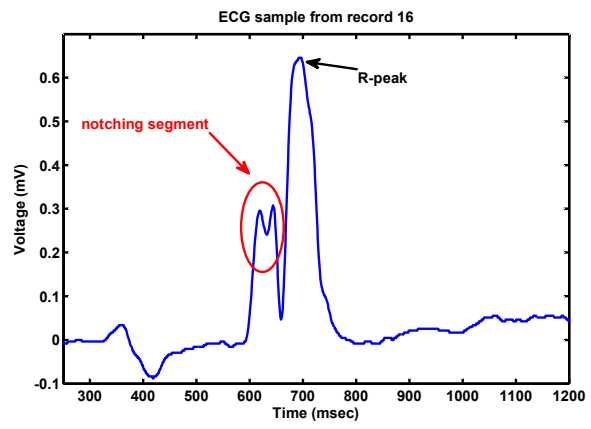

(a) An example of two notches in the QRS

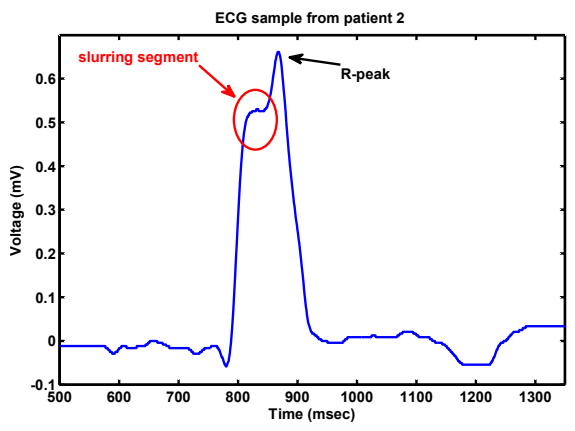

(b) An example of slurring in the QRS

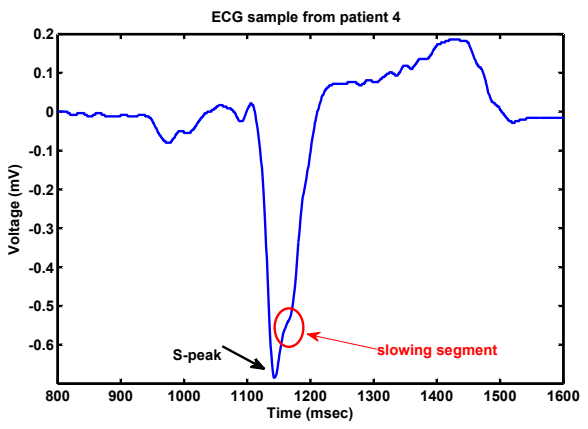

(c) An example of slowing in the QRS

Fig. 1. The three types of fractionation

of the scar size, by relying only upon the widely available ECG signal, the cost savings compared to its detection and quantification through costly imaging techniques, like CMR, could be significant. Although attempts have been made to automate the original version of the Selvester scoring model, which does not include any confounder determination and in some cases not even fractionated QRS, no published data demonstrate the automation of the updated, modified score [7]-[9], which is more relevant to the clinical practice.

The primary objective of the present work is to develop an automated Selvester scoring system based on the updated Selvester score, for the quantification of myocardial scar from 12-lead ECG. To our knowledge this is the first of its kind. The most challenging part of the updated Selvester score is the identification and characterization of fractionated QRS segments, which is required for confounder classification and awarding points. It is common, even for well-trained cardiologists, to misdetect or misclassify QRS fractionations, due to the variability of fractionated QRS, when manually inspecting the ECG trace. On the other hand the evolution of Signal Processing methods, during the previous decade, provided various techniques for the automated analysis of the ECG signal [10]. In this work, we propose a novel algorithm for the automated detection and pattern identification of fractionated QRS segments, based on the Stationary Wavelet Transform (SWT), which alleviates the problems of manual fractionation detection and provides a tool for in-depth analysis of fractionated ECG. In our approach, the operations of detecting fractionated QRS and ascertaining its morphology, classification of the confounders and the subsequent scoring mechanism are all integrated under one framework, resulting in an end-to-end realization of the automated updated Selvester scoring system which, with no human intervention, can compute the Selvester score in a fast and accurate manner. In our design we follow the definitions of fractionation as provided in [6]. To evaluate the performance of the developed system, standard 12-lead ECG recordings were obtained from patients known to have ischemic heart disease, enrolled in a study at the University Hospital Southampton NHS Foundation Trust. The confounder classification and Selvester scores were manually calculated by expert cardiologists for all records and then were compared with the same as calculated by the proposed automated system. The results show that for classifying the confounders, the automated system agrees to the manual classification in $94 \%$ (48 out of 51 records) of the cases. Similar accuracy (47 out of 51 records) was also observed in the computation of the Selvester score by the automated system, within 1-point of the manual scoring. These results show that the proposed system exhibits clinically acceptable performance.

The rest of the paper is structured as follows: in Section II we provide a brief background of the updated Selvester score and the definitions of fractionations in the QRS. Section III describes the algorithmic development of the automated updated Selvester scoring system including a novel algorithm for detecting fractionations in the QRS-complex and ascertaining the fractionated QRS morphology and confounder class. In Section IV we present experimental results for validating the developed system while conclusions are drawn in Section V.

\section{BACKGROUND}

\section{A. Updated Selvester Scoring System}

The updated Selvester QRS score is a 50-criteria/31-point method for approximating scarring in the left ventricle from ECG recordings. The scoring criteria are selected according to the conduction pattern of the ECG, in order to take account of features which are part of common disease processes but might be mistaken for those representing scar. Such confounding categories considered are left bundle branch block (LBBB), right bundle branch block (RBBB), left anterior fascicular block (LAFB), left ventricular hypertrophy (LVH), $\mathrm{RBBB}+\mathrm{LAFB}$, or a normal pattern. The presence of these is determined according to the electrical axis of the heart, various QRS width and amplitude measurements and morphological features, including the QRS pattern and the presence of fractionations in the QRS seen as notching, slurring or slowing. In addition, the criterion thresholds are adjusted to account for changes on the ECG due to right atrial overload (RAO)/right ventricular hypertrophy $(\mathrm{RVH})$ (defined by a P-wave amplitude in $V 1 \geq 0.1 \mathrm{mV}$ or in $a V F \geq 0.175 \mathrm{mV}$ ), and for younger men with increased voltage and older women with lower voltage. The ECG leads considered in the scoring rules are I, II, aVL, aVF, and V1 through V6 and points are then awarded according to the presence of fractionations in QRS and various durations and amplitude criteria [6]. Each Selvester score point represents $3 \%$ of myocardial scar. The detailed description of the scoring scheme including the confounder determination 
is described vividly in [6] and therefore is omitted here for brevity.

\section{B. Fractionated $Q R S$}

Fractionations in QRS result from delayed conduction and typically are visible in three forms: notching, slurring and slowing. Notching is defined as a reversal in the QRS trace with an angle $>90^{\circ}$ and amplitude $>0.05 \mathrm{mV}$. As an example, Fig. 1(a) shows an R-wave where two notches are present. Contrary to notches, slurs are segments where an ECG signal exhibits minuscule change in amplitude between successive samples, thereby creating a "plateau"-like segment. Fig. 1(b) shows an example of slurring in an R-wave. The third component of fractionation, slowing, is characterised by an almost smooth declined rate of change in the QRS waveform. Although it is closely similar to slurring, the major difference is that slowing segments have a finite gradient (angle $<90^{\circ}$ ) compared to the "plateau"-like nature of slurring. Fig. 1(c) shows an example of slowing in the S-wave.

It is to be noted that fractionation may occur in any of the three waves $(\mathrm{Q}, \mathrm{R}$ and $\mathrm{S})$ of the $\mathrm{QRS}$-complex resulting into a number of different morphologies of fractionated QRS, e.g. RS, RSR', RSR'S' etc., where by definition, the preceding positive/negative deflection is characterised as R-/S'-peak and the succeeding as R'-/S'-peak. Two such morphologies (RS' and RSR'S') are shown in Fig. 2 as an example. Successful determination of these typical patterns is of high importance for the Selvester scoring system as the overall fractionated QRS patterns are used both for confounder classification as well as for awarding points.

\section{Algorithmic FORMUlation}

The algorithmic flow of the proposed automated Selvester scoring system is depicted in Fig. 3. It consists of four main parts - isoelectric line detection and QRS wave segmentation, fractionated QRS detection and its morphology characterisation, and the implementation of the actual scoring scheme. The actual scoring can be divided into two parts, viz. confounder classification and the point awarding procedure. In the following subsections each of these blocks is explained in details. In the proposed algorithm the only inputs are the ECG signal itself (one PQRST-complex) and the temporal locations of the P-onset and P-offset, if the P-wave appears on the ECG signal as well as the QRS-onset and QRS-offset.

\section{A. ECG Database}

In our investigation we used 51 standard 12-lead ECG records from patients enrolled in a study at the University Hospital Southampton NHS Foundation Trust. All subjects underwent examinations particularly for the detection of myocardial scar through elaborate medical tests (i.e. CMR), thus there was unambiguous knowledge on the presence and extent of scarring which is the paramount requirement for validating the present work as in essence, the Selvester score tries to estimate the extent of scarring from the ECG. Such a cohort provides a suitable database for the formulation of our fractionation detection algorithm and the automated Selvester scoring system. However the digital ECG recordings of the 51 subjects could not be utilized directly due to proprietary encryption applied by the manufacturer of the acquisition device. We therefore opted to employ the ECGScan software, clinically validated in [11], for its ability to reconstruct a digital ECG from printed paper ECGs, in order to manually digitize the 51 signals used in our analysis from printed paper ECGs at a rate of 1000 samples/s. The signal quality of the digitized ECGs was carefully examined and uniformly endorsed, from a clinical perspective, by three practicing expert cardiologists, at the very beginning of our experimentation. As a second level of verification, the digitized ECGs were also used by the cardiologists in the manual calculation of the Selvester score (see Section IV). The $1 \mathrm{KHz}$ digitization rate was chosen in order to have sufficient resolution in capturing the abrupt changes in the direction, thus the monotonicity of the ECG trace in fractionated QRS segments. Considering the experience of the cardiologists, it is imperative to employ such a high sampling frequency for detecting and classifying fractionated QRS segments unambiguously. In addition, latest generation 12-lead ECG machines, both hospital-based and ambulatory, are capable of acquiring the ECG signal with such high sampling frequencies (even higher than $1 \mathrm{KHz}$ ).

\section{B. Isoelectric line detection and segmentation}

The detection of the isoelectric line of the PQRST complex is critical here since it is used as the reference for calculating the amplitudes of ECG peaks. In principle, we follow the same method described in [12] for its extraction, where two median filters are used with time window of 200 and $600 \mathrm{~ms}$. In addition, we use a third median filter of time window (P-offset - QRS-onset) only when the P-wave is present. The output of the series of median filters is considered as the extracted isoelectric line.

Once the isoelectric line is detected we apply it for determining the different waves present in the QRS-complex by finding out the intersection points of the isoelectric line and the QRS-complex as it is localized by the QRS boundaries, used as inputs. The QRS-onset and QRS-offset are considered as the beginning instance of the first wave and the ending point of the last wave respectively and excluded from the intersection investigation. The process is depicted pictorially in Fig. 4 for the two samples of Fig. 2. If two consecutive intersection points occur within $5 \mathrm{~ms}$, only the first one is considered as the other is attributed to local fluctuation. In addition an amplitude rule, which stipulates that the peak of each detected wave, defined by two successive intersection points should demonstrate higher than $\pm 0.009 \mathrm{mV}$ separation from the isoelectric line was applied, following the cardiologists directives. If this is not satisfied, the detected interval is again attributed to local fluctuation and the latter intersection point is discarded. In this process although theoretically we should have 2 intersection points, since sometimes one or two of the waves may not be present, the occurrence of less than 2 intersection points is normal. Conversely, in the presence of fractionated segments (see Fig. 4(b), additional intersection 


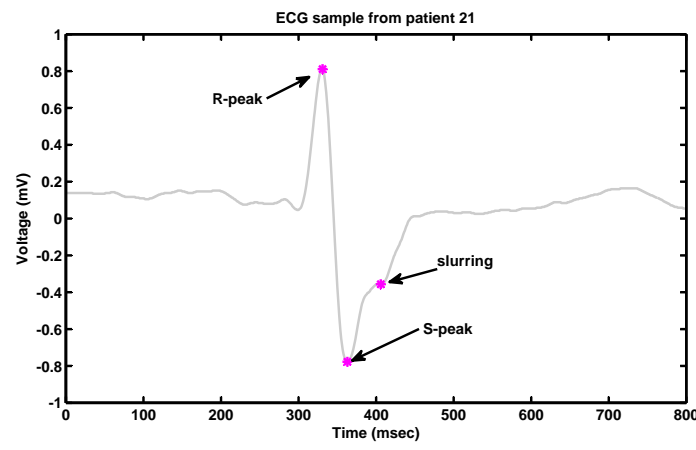

(a) RS pattern with slurring on the S-wave

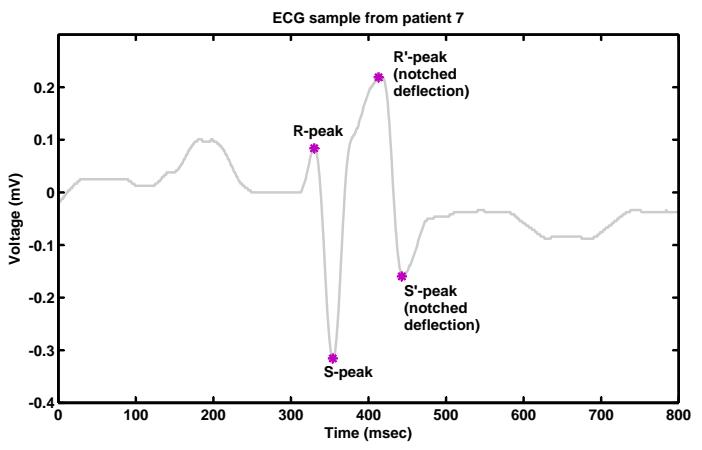

(b) RSR'S' pattern with additional deflections (notches)

Fig. 2. Two examples of fractionated QRS pattern

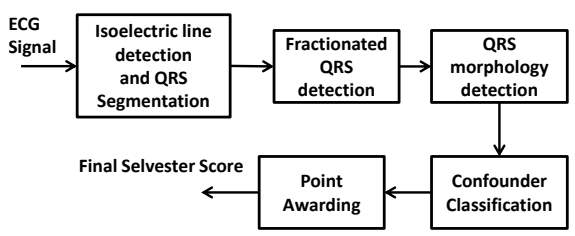

Fig. 3. Block diagram of the Automated Selvester Scoring System

points may be encountered. However, in all cases between a pair of intersection points we follow the clinical definitions of $\mathrm{Q}, \mathrm{R}$ and $\mathrm{S}$ waves based on the polarity of the deflections and their characteristic sequence of occurrence, in identifying them individually.

\section{Detection of fractionation in the QRS-complex}

Over the years the time-frequency localisation property of Discrete Wavelet Transform (DWT) with various forms of mother wavelet has been used successfully for detecting the temporal positions of the constituent waves of an ECG [13][15]. It is in principle a cascaded structure of filters where the transfer function of each of them is determined by the mathematical form of the mother wavelet used. In essence, it decomposes an input signal into different resolution scales by computing two vectors of detailed (cD) and approximate (cA) coefficients respectively. In our work we selected the Haar function as the mother wavelet. According to the definition of the Haar function, the cD coefficients generated, at each DWT resolution scale, are proportional to the derivative of the local averages of the input which is a filtered version of the original signal [16]. This is the main feature of DWT that is used in detecting the various waves in an ECG. Thus any peak occurring in an ECG time series is manifested as a pair of maxima-minima (sign change) and a zero-crossing or zerovalue point between them in the $\mathrm{cD}$ space [14], [17]. This fundamental principle allows one to explore the monotonicity of a given signal by applying Haar DWT. Since the three classes of fractionations described in Section II are in essence deviations from monotonicity, one may use the Haar DWT for detecting QRS fractionations, through exploration of the $\mathrm{cD}$ coefficients value and characterising them. In principle other mother wavelets (e.g. quadratic spline) share the same derivative-proportionality property as Haar, in this respect and therefore can also be adopted for analyzing the monotonicity of the ECG signal. However, Haar wavelet is the simplest form of wavelets from a computational complexity point of view. Subsequently in our work we opted to choose the Haar function as the mother wavelet. In addition, since the DWT maintains the temporal resolution of the input signal, the monotonicity deviations can also be localized in time. Nevertheless, conventional DWT suffers from the fact that at each level of decomposition the number of input samples is reduced to half and therefore higher decomposition scales suffer from reduced time resolution. The Stationary Wavelet Transform (SWT) is a class of DWT that eliminates this problem and achieves a time-invariant decomposition by removing the subsamplers after each filtering operation and interpolating the impulse response of the filters [18]. Therefore for detecting the presence of fractionations in the QRS-complex we have used the SWT with the Haar function, as the main analysis method.

Although in our fractionation detection algorithm, we predominantly adopted the clinical definition of the different types of QRS fractionation [6], it is possible, due to local fluctuations, to misdetect a type of fractionation as another. The detection of notching due to its distinct morphology is less vulnerable to this effect, but because of the possibly similar morphology of slurring and slowing, misclassifications may occur in the presence of local fluctuations. To mitigate this effect our strategy is to follow the expert decisions of practicing cardiologists. Based on their opinion we have empirically formulated a set of rules for distinguishing the slowing segments, in particular from slurring. To achieve this, three expert cardiologists first annotated the fractionations (notching, slurring and slowing) in 10 ECG signals chosen by them as representative examples that exhibit extreme types of fractionations and more specifically when slurring and slowing segments appear to be quite similar. During the annotation they were blind to the algorithmic outcome. The algorithmic outcomes were then validated against the manual annotations for these 10 ECGs and were found very much accurate particularly for notching and slurring. On the other hand, slowing segments, because of their close similarity to slurring, were sometimes detected as slurs. We therefore tuned the set of rules for the detection of slowing, using the manual annotations of the cardiologists for the $10 \mathrm{ECG}$ 


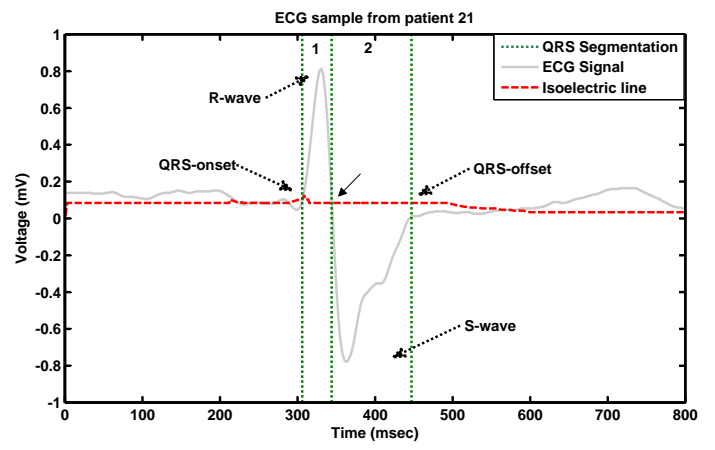

(a) RS-pattern (2 deflections)

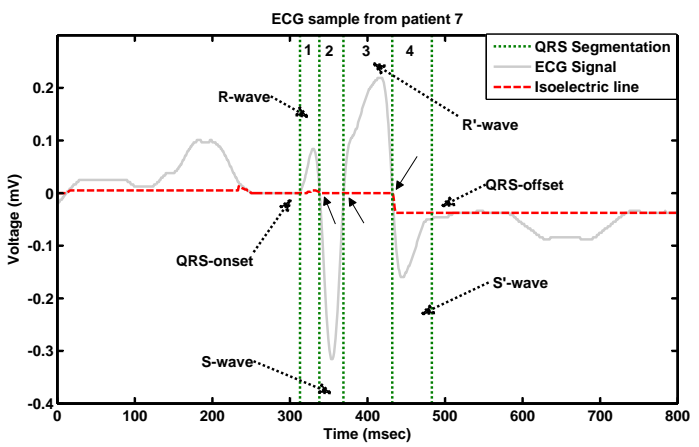

(b) RSR'S'-pattern (4 deflections)

Fig. 4. Identification of the QRS morphological pattern after the extraction of the isoelectric line. The solid arrows denote the intersection points between the isoelectric line and the QRS trace. The QRS waves are also numbered

signals. In the following sections we analyze in details the rules we formulated for the detection and classification of the three types of QRS fractionation. The practical soundness of these rules is ultimately assessed during the evaluation of the proposed automated updated Selvester scoring system (in Section IV) and particularly in the system's ability to accurately categorize testing records in the correct confounder group.

The isoelectric line compensated and segmented ECG signal is first subjected to SWT analysis up to $2^{4}$ resolution scale and then we focus entirely on the $\mathrm{cD}$ coefficients of the already localised QRS-complex. The coefficients of the $2^{1}$ resolution scale were discarded from our analysis since it is already established (which also agrees to our observation) that this scale is mainly dominated by high-frequency noise components [13]. Therefore we concentrate in analysing the $\mathrm{cD}$ coefficients of the remaining resolution scales $\left(2^{2}, 2^{3}\right.$ and $\left.2^{4}\right)$.

1) Extraction of notching: Following the basic principle of monotony deviation detection, presented at the beginning of this section, in the $\mathrm{cD}$ space of a resolution scale, a potential notch in a QRS-complex is represented as two local maximaminima pairs with a zero-crossing point between each of them. This is because, in a notch the direction change of the trace occurs twice (see Fig. 1(a)), hence each maxima-minima pair corresponds to each change in direction. On the other hand, the main peak of each one of the QRS waves is expected to be represented by one pair of maxima-minima since the direction change occurs only once. This distinction is crucial as it enables one to classify notches and main peaks accurately.

We start the analysis by detecting all the zero-crossing points in the $\mathrm{cD}$ space of resolution scale $2^{2}$ (cD_12), thus localising each of the maxima-minima pairs present at that scale. For each zero-crossing point in $\mathrm{cD}$ _12 located between $c D$ coefficient number $j-1$ and $j$, we investigate whether there exists a zero-crossing point between $\mathrm{j}-3$ and $\mathrm{j}-1 \mathrm{cD}$ coefficients in resolution scale $2^{3},\left(\mathrm{cD} \_13\right)$. The reason behind this process is that it is established that the high-frequency components (like a notch) of an input signal are mainly localised within the scales $2^{2}$ and $2^{3}$. Therefore if a zero-crossing point is detected in both of these resolution scales corresponding to the same time-indexed position of the signal then it can be concluded that a valid notch is present and this is not a noise based fluctuation. The difference of the indices used for the cD space search window, in $2^{2}$ and $2^{3}$ scales is related to the inherent delay of the SWT computing process and therefore the windows position is adjusted accordingly. The process for detecting notches in the QRS is depicted in Fig. 5. To eliminate potential notches present on the isoelectric line (which are of no clinical significance), we measure the amplitude of each of the detected notches with respect to the isoelectric line and those with amplitudes within $\pm 0.015 \mathrm{mV}$ of the isoelectric line are disregarded as they are considered to be isoelectric line fluctuations. The rest of the detections are retained as valid notches.
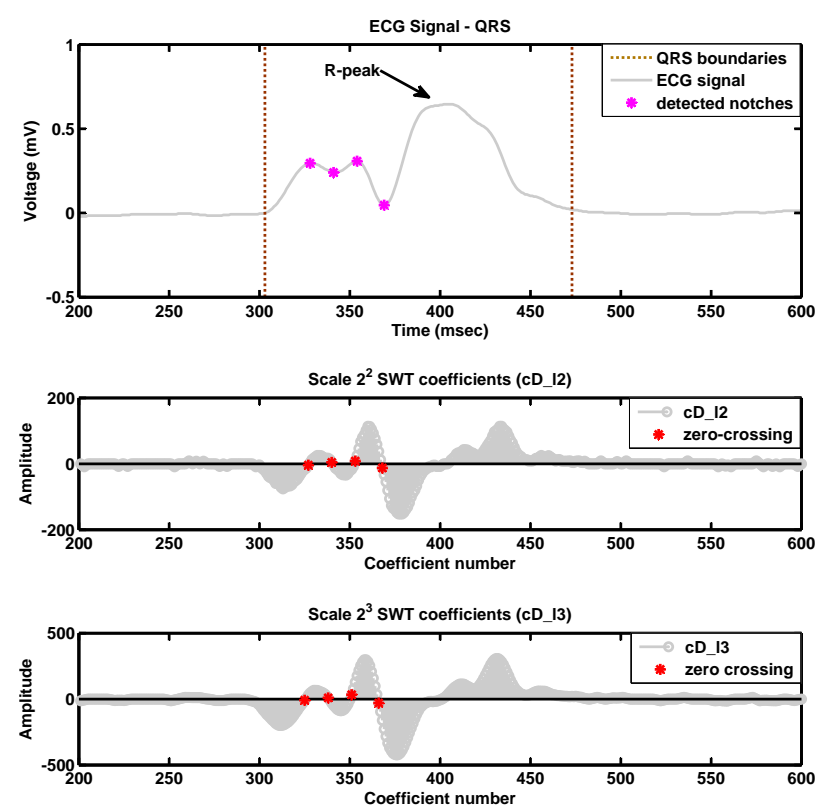

Fig. 5. Detection of notches in the signal of Fig. 1(a)

2) Extraction of slurring: As mentioned in Section II, slurring is characterised by a "plateau"-like segment in the QRS-complex. Therefore according to the principle described earlier for $\mathrm{cD}$ coefficient computation, a slurring segment in the QRS-complex manifests itself as a sequence of consecutive zeros in the $\mathrm{cD}$ space. The analysis for detecting consecutive zeros is carried out in the $\mathrm{CD}_{-} 12$ space. If a sequence of two 
or more consecutive zeros is detected in the cD_12 space a slurring segment is identified with its beginning and ending (in time) coinciding with the first and last zero of the sequence in the cD_12 space. However, in practice we observed that even within a clinically accepted slurring segment (as identified by expert cardiologists) local fluctuations may occur which may result in a little deviation from a strict "plateau"-like morphology. This phenomenon is reflected in the cD_12 space as two or more sequences of consecutive zeros with finite values of $\mathrm{cD}$ _12, in between them. Since they are still clinically considered as part of the same slurring segment, during the analysis we adopted the strategy that if two or more sequences of consecutive zeros occur within $8 \mathrm{~ms}$ of each other then they are considered as part of the same slurring segment. This time window is selected both by observation and in consultation with the expert cardiologists. In this case, the time position corresponding to the first zero of the first sequence in $\mathrm{cD}$ _12 space is considered as the beginning of the slurring segment and the last zero in the last sequence is considered as the end point of it.An example of this procedure is shown in Fig. 6 where two sequences of more than two consecutive zeros occur within the same slurring segment. However since their separation is less than $8 \mathrm{~ms}$, they can altogether characterise the beginning and the end of a single slurring segment. Similar to the notching fractionation, once a slurring segment is detected, its amplitude with respect to the isoelectric line is measured and if it is found within $\pm 0.015 \mathrm{mV}$ of the isoelectric line value, it is disregarded and attributed as part of the isoelectric line itself. This process eliminates any slurringlike segments towards the end of a QRS-complex when the ECG trace gradually returns to the isoelectric line-a typical phenomenon observed in a number of leads.
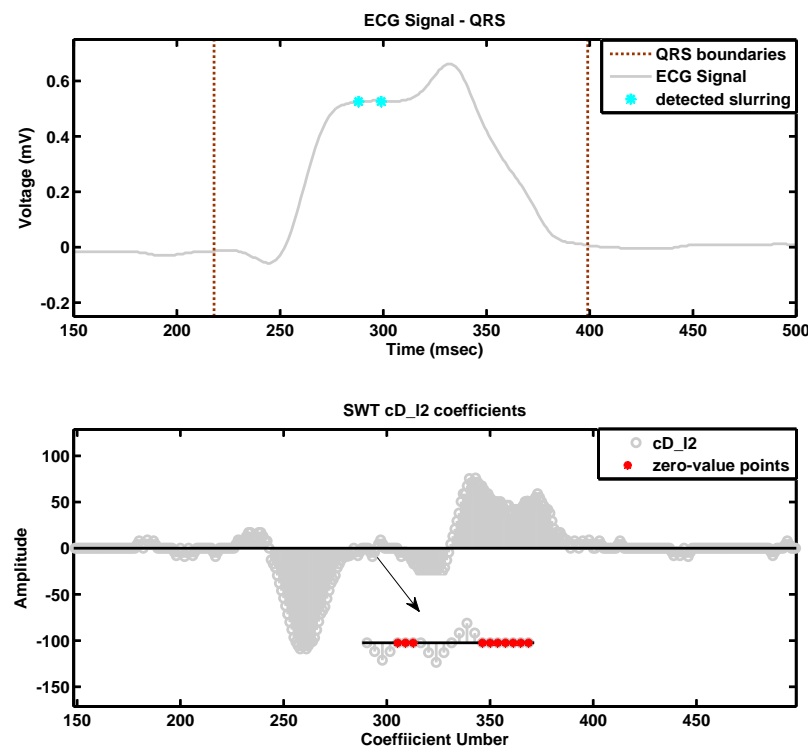

Fig. 6. Detection of slurs in the signal of Fig. 1(b)

3) Extraction of slowing: Unlike the notching and slurring segments where due to their specific morphologies a one-toone mapping exists between the $\mathrm{cD}$ space and time series data, no one-to-one mapping of that kind exists for slowing segments. This is due to the fact that slowing is defined as a change of the rate of the ECG trace that is neither a sudden change in direction of $\geq 90^{\circ}$ (like notch) nor a "plateau" (like slurring). Therefore we have employed an empirical method based on experimental observation for detecting the slowing segments as described in the following. We have experimentally observed over 51 ECG records from ischemic patients, that when a clinically identified slowing segment is present, the corresponding $\mathrm{cD}$ coefficients show at least 3 local extrema between two consecutive zero-crossing points (or zero-value points) at the $2^{4}$ resolution scale (cD_14). This directly implies that in the gradient space of $\mathrm{cD}_{-} 14$, at least 3 zero-crossing points (each one corresponding to an extrema in cD_14) will exist. Having detected all the zero-crossing and zero-value points as part of the extraction of notching and slurring, we translate the intervals between them to the cD_14 space by appropriately shifting them and consequently define a search window within which the gradient $\left(\operatorname{grad}_{\mathrm{cD}} 14\right)$ of $\mathrm{cD} \_14$ is computed. This enables us to compute the number of zerocrossings, in $\operatorname{grad}_{\mathrm{CD}_{1} 14}$ within each of those intervals. The $\operatorname{grad}_{\mathrm{cD} \_14}$ is approximated by calculating the forward difference on the two edges and the centred difference in the interior points of each window. Let us consider, as an example (as shown in Fig. 7) that we have two zero-crossing (or zerovalue) points in $\mathrm{CD} \_14$ at positions $z_{1}$ and $z_{2}$ and the gradient of cD_14 exhibits at least 3 zero-crossing points (according to our observed criterion) within that interval at positions $q 1, q 2, q 3$. We then first extract the cD_14 coefficient with maximum absolute value $(\mathrm{M})$ within $\left[z_{1}, z_{2}\right]$. Since $z_{1}, z_{2}$ are sequential zero crossings the value of cD_14 will not change sign between these two points. Following we check the sign of the gradient in $\left[q_{1}, q_{2}\right]$ and if found positive/negative we extract the maximum/minimum value of $\operatorname{grad}_{\mathrm{cD} \_14}$ in $\left[q_{1}, q_{2}\right]$ and the minimum/maximum value of $\operatorname{grad}_{\mathrm{CD}_{-} 14}$ in $\left[q_{2}, q_{3}\right]$ denoted as A1,A2 in Fig. 7 respectively. If there are more than three zero-crossing points in $\operatorname{grad}_{\mathrm{cD}} 14$ (as it is the case in Fig.7), we consider only an odd number of zero crossings and extract either the maximum or the minimum value of $\operatorname{grad}_{\mathrm{cD} \_14}$ in each subsequent window defined by the number of zero-crossing points, based on the sign of the $\operatorname{grad}_{\mathrm{CD}_{-} 14}$ within that window. If there are more than three zero-crossing points in $\operatorname{grad}_{\mathrm{cD} \_14}$ then accordingly there will be additional pairs of maxima-minima. We then compare each of these pairs $(|A 1|,|A 2|$ and $|A 3|,|A 4|)$, starting with the one with highest absolute sum of amplitudes $(|A 1|+|A 2|>|A 3|+|A 4|)$, to the absolute value of $\mathrm{M}$ according to the relations stated in Eq. 1 and if both of these are satisfied a slowing fractionation is detected and localised in the middle zero-crossing point $\left(q_{2}\right.$ in our example), from the three $\left(q_{1}, q_{2}, q_{3}\right)$ zero crossings used to define the search windows of $\mathrm{A} 1, \mathrm{~A} 2$ in $\operatorname{grad}_{\mathrm{CD}} 14$.

$$
\begin{aligned}
|A 2| & >0.01 M \\
|A 1|+|A 2| & >0.075 M
\end{aligned}
$$

\section{Extraction of the QRS morphology pattern}

Based on the presence of fractionation in the QRS-complex, as detected by the methods described in the foregoing subsections, one needs to determine the overall fractionated 

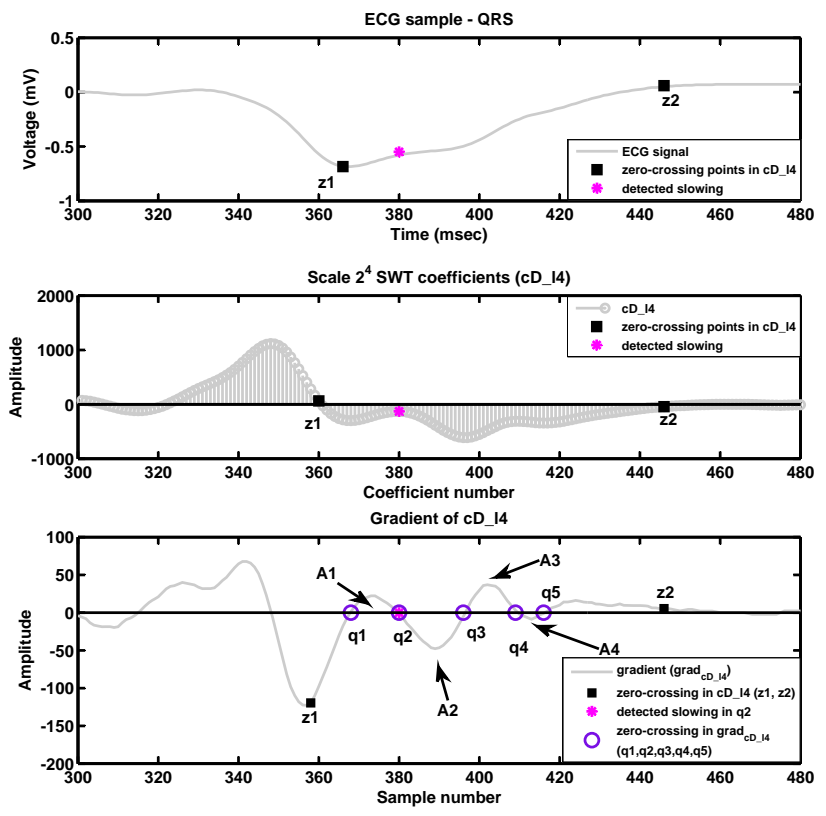

Fig. 7. Detection of slowing in the signal of Fig. 1(c). Squares indicate the zero-crossing points in $\mathrm{cD}_{-} 14(z 1, z 2)$, circles indicate the zero-crossings in $\operatorname{grad}_{\mathrm{cD} \_14}(q 1, q 2, q 3, q 4, q 5)$ within $[z 1, z 2]$ and the star that coincides with $q 2$ indicates the detected slowing fractionation after applying Eq.1

morphology of the QRS-complex in order to proceed for confounder classification and point awarding as mentioned in Section II-B. Note that the fractionation detection algorithms used here, in essence detect the deviation from monotonicity in an ECG trace. Therefore it is intuitive that within the pool of "detected" fractionations even the non-fractionated (normal) $\mathrm{Q}, \mathrm{R}$ and S peaks will also exist, since morphologically at the peaks, these waves also exhibit deviation from monotonicity. Fig. 8 shows an example where a clinically accepted nonfractionated R-peak is identified as a slurring segment since the rate of change of the ECG trace at the peak point is small, thus creating a small "plateau"-like segment. In addition, in several cases, the same fractionation segment could be detected as more than one type depending on their local fluctuation. To eliminate erroneously detected fractionation segments and thereby extract the true fractionated QRS morphology a set of empirical rules based on clinical observations and definitions has been applied and described as follows:

- If 2 fractionations are detected within $10 \mathrm{~ms}$ of each other, they are considered as one and the latter type is retained

- If 3 or more fractionations are detected in a wave interval, then the normal wave peak is considered present alongside one or more fractionation segments

- Detected fractionations within $6 \mathrm{~ms}$ of the QRS boundaries are ignored

- Of all the detected fractionations within a wave interval ( $\mathrm{Q}, \mathrm{R}$ and $\mathrm{S}$ etc..) the one which exhibits highest separation from the isoelectric line is labeled as the actual wave peak and in the case of only one fractionation detected, this is classified as the main peak in that wave labeled as $\mathrm{Q}, \mathrm{R}$ or $\mathrm{S}$ peak

- Detection of 2 fractionations within a wave, indicates that either slurring or slowing fractionation exists along with the main peak in that wave

- Each notch will always be associated with 2 fractionations detected. Of the pair of points the notch is localised based on the deflection direction of the wave. If the wave is positive (R-wave) the maximum of the two is used, conversely if it is negative (Q-wave, S-wave) the minimum one is considered as the notch

- Slowing fractionations do not correspond to main wave peaks

Based on these rules the presence of fractionation segments will define the actual fractionated QRS morphology.
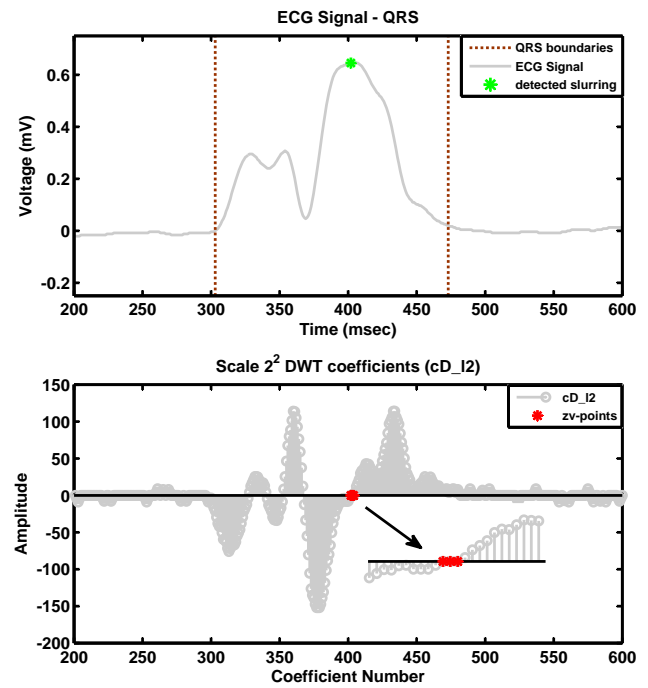

Fig. 8. Detection of the R-peak of Fig. 1(a) as slur

\section{E. Confounder classification and point awarding}

Once all the fractionated segments and the final QRS morphological pattern is extracted, they are applied for confounder classification and subsequently for awarding points. The basic rules for these purposes are shown in the flowchart of Fig. 9 which follows, without any modification the directives established by Loring et. al. and has been reproduced from Fig.2Fig.4 which appear in [6]. For brevity, the specific rules for each confounder category are not listed here, but they were implemented exactly as they appear in Fig.5 of [6]. As can be seen from Fig. 9, one needs to compute the electrical axis of the heart in order to invoke the entire process.

Typically all the electrical signals (depolarizations) generated from the heart can be represented as a vector and its orientation from the origin is considered as the electrical axis of the heart. Deviation or changes from the normal axis values may be indicative of pathological conditions. The conventional way of calculating the heart's electrical axis is to identify the isoelectric lead - the lead whose net deflection (R_ampl - S_ampl - Q_ampl) is closer to the isoelectric line. The heart's electrical axis is then calculated as perpendicular to the orientation of that lead. However it is also recognised that this definition has its own shortcomings and therefore following the directives of expert cardiologists we adopted a modified way for identifying the electrical axis as described below [19], 


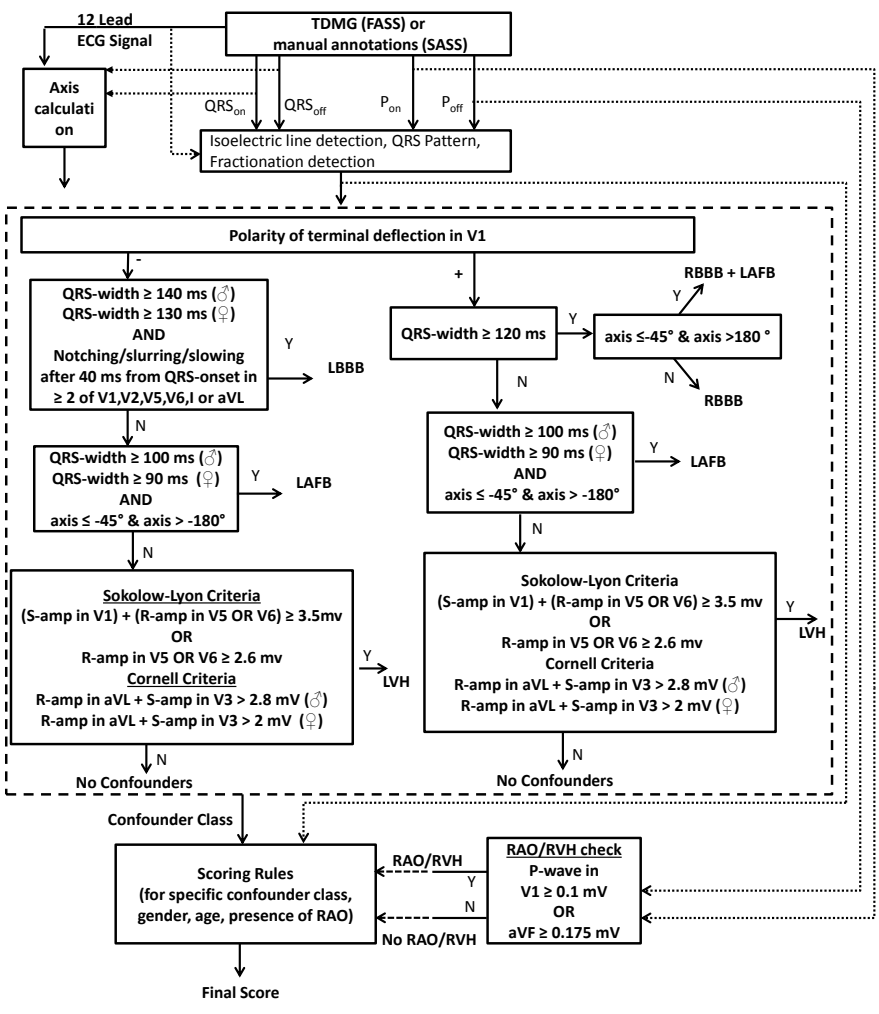

Fig. 9. Flowchart of the Automated System. Reproduced according to Fig.2Fig.4 from [6]

[20]. Firstly, the Area under the Curve (AUC) is computed using trapezoid rule for the QRS-complex of the limb leads (I, II, III, avL, avF and avR). The QRS boundaries and the derived isoelectric line are used as reference for the AUC computation. Finally, these AUCs are used for computing the heart's electrical axis from the following equations, where $A \#$ denotes the AUC of lead \#.

$$
\begin{aligned}
& x_{\text {axis }}=A_{I} \cos \left(0^{\circ}\right)+A_{I I} \cos \left(60^{\circ}\right)+A_{I I I} \cos \left(120^{\circ}\right)+ \\
& A_{a v R} \cos \left(-150^{\circ}\right)+A_{a v L} \cos \left(-30^{\circ}\right)+A_{a v F} \cos \left(90^{\circ}\right) \\
& y_{\text {axis }}=A_{I} \sin \left(0^{\circ}\right)+A_{I I} \sin \left(60^{\circ}\right)+A_{I I I} \sin \left(120^{\circ}\right)+ \\
& A_{a v R} \sin \left(-150^{\circ}\right)+A_{a v L} \sin \left(-30^{\circ}\right)+A_{a v F} \sin \left(90^{\circ}\right) \\
& \angle a x i s=\left\{\begin{array}{c}
\left(\frac{360^{\circ}}{2 \pi}\right) \tan ^{-1}\left(\frac{y_{\text {axis }}}{x_{\text {axis }}}\right), A_{I}>0 \\
-180^{\circ}+\left(\frac{360^{\circ}}{2 \pi}\right) \tan ^{-1}\left(\frac{y_{\text {axis }}}{x_{\text {axis }}}\right), A_{I}<0, A_{\text {avF }}>0 \\
180^{\circ}+\left(\frac{360^{\circ}}{2 \pi}\right) \tan ^{-1}\left(\frac{y_{\text {axis }}}{x_{\text {axis }}}\right), A_{I}<0, A_{\text {avF }}<0
\end{array}\right\}
\end{aligned}
$$

Once the electric axis of the heart is computed we follow the rules described in Fig. 9 for confounder classification and awarding points. The individual Selvester scoring point rules for each confounder class are described in details in [6]. As mentioned, the developed system requires different ECG parameters as its principal inputs including the P-onset/offset and the QRS-onset/offset in addition to the calculated electrical axis. For extracting these ECG features and various measurements of ECG waves (like amplitudes and durations) we have previously proposed an automated algorithm that extracts the ECG features with high accuracy using time domain morphology analysis principles (termed as TDMG algorithm) [21]. Therefore this TDMG algorithm can be integrated with the automated Selvester scoring algorithm proposed here to develop a self-contained fully automated system, henceforth termed as the Fully Automated Selvester Scoring system (FASS). On the other hand, manually annotated values of the required ECG parameters could be used as the primary inputs of the automated Selvester scoring system proposed here, resulting in a semi-automated Selvester scoring system (SASS). Both these scenarios are depicted in Fig.9

\section{RESUlTS AND Discussion}

\section{A. Formulation of the evaluation standard}

To formulate the gold standard for evaluating the performance of our system, three cardiologists independently classified the confounder group and computed the updated Selvester score for each of the 51 ECG records in manual fashion, following the clinical standard procedure of the updated Selvester score calculation [6]. The manual scores and confounding categories, from each annotator, were then compared to each other and whenever a disagreement was found a consensus among the three annotators was reached. In the end of this process, a single consented confounding category and manual score was considered for each record which was then used as the gold standard for validating the outcomes of the automated system. In parallel with the manual calculation, the confounding category and the Selvester Score were derived for each of the 51 records from the developed automated system as explained in the following. During this process, the cardiologists were blind to the results of the automated system.

\section{B. Testing strategy}

We employed an integrated testing strategy for the proposed system. This strategy involves two steps of validation - accuracy of the confounder classification and accuracy of the actual scoring. In order to isolate any erroneous effects originating from the automated ECG parameter extraction, using the TDMG algorithm, that may affect the final performance of the complete system we run two different validation experiments - one with the FASS system and the other with the SASS system, where the input ECG parameters (P-onset/offset, QRSonset/offset) are manually annotated by the expert cardiologists. In addition, when doing the validation experiment with the FASS system, in 26 records (half of the total records) we chose to calculate the Selvester score from a different heartbeat than the ones used in the SASS system, covering a wider range of heartbeats for the system evaluation. This enables us to make a consistency check for the performance of the FASS and SASS systems when: 1) the same heartbeats are used in both cases (25 cases) and, 2) different heartbeats are used (26 cases) for the two systems.

1) Validation of the Confounder classification: Fig. 10 shows the results of the confounder classification by the SASS (Fig. 10(a)) and the FASS (Fig. 10(b)) systems compared to the manually inferred confounders by the cardiologist team. Out of 51 records, the SASS system is able to classify the 


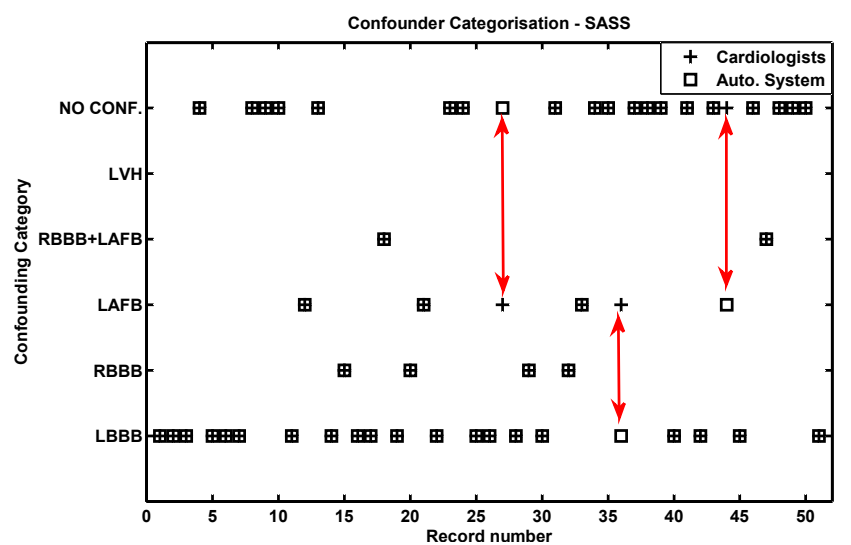

(a) SASS

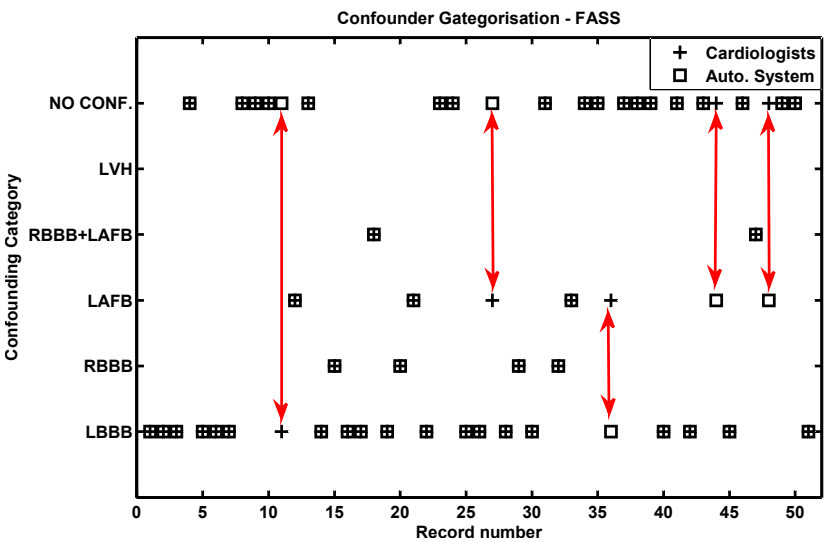

(b) FASS

Fig. 10. Cardiologists and Automated System Confounder categorization results

confounders correctly in 48 records $(94.1 \%)$ whereas the FASS system does the same in 46 records (90\%). Table I lists the individual records that exhibited classification discordance compared to the manual classification. Records 27, 36 and 44 were classified differently by both the FASS and SASS systems whereas records 11 and 48 are additionally misclassified by the FASS system. In records 27, 44 and 48 the disagreement results from the derived value of the hearts electrical axis. Table II illustrates the differences in the axis calculations in these three cases. In record 27 this was caused due to the extremely irregular shape of the ECG waveform on the avR lead which affected the calculation of the axis from Eq. (3) resulting in a value $\left(-23^{\circ}\right)$, for both SASS and FASS systems, that diverges significantly from the cardiologists value $\left(-90^{\circ}\right)$. In record 44 the axis angle was found to be $-45.4^{\circ}$ (FASS) and $-57^{\circ}$ (SASS) instead of $-30^{\circ}$ provided by cardiologists. This was caused due to significant isoelectric line wandering which affected its approximation from the automated systems, thus the value of the AUC in Eq. (3) and ultimately the axis calculation. With these axis values, both for SASS and FASS, the LAFB criteria are satisfied, thus the record is misclassified by the automated systems as LAFB, instead of the no confounders class given by cardiologists. It must be noted than the axis calculation in FASS will also be affected by the QRS boundaries provided by the TDMG algorithm. This was the case in record 48 , where the annotators value was $-30^{\circ}$ and the automated system's value was $-43^{\circ}$ and $-47^{\circ}$ for SASS and FASS respectively. The classification discrepancy was present only in the case of FASS where the axis value satisfied the LAFB criterion (axis $\leq-45^{\circ}$ ). This was caused due to inaccurate QRS boundaries obtained by the TDMG algorithm.

The misclassification of record 36 is attributed to a less accurate approximation of the baseline due to ST elevation. This resulted into the terminal deflection (the last segment of the QRS) to be classified as a negative deflection instead of a positive deflection and a valid notch to be disregarded due to the amplitude criterion, hence leading to the confounder disagreement. Finally record 11 was classified differently by the FASS system because of the QRS-onset value computed by the TDMG algorithm, which resulted in a notching fractionation to be disregarded. According to the manual annotation the notch was detected $41 \mathrm{~ms}$ after the onset of the QRS, whereas with TDMG the same notch was detected $39 \mathrm{~ms}$ after the QRSonset. Since the updated Selvester scoring system stipulates that only fractionations located after $40 \mathrm{~ms}$ of the QRS-onset are considered valid, the detected notch in the former case was accepted as a valid notch, whereas it was disregarded in the latter, even though the misdetection is only by $2 \mathrm{~ms}$. These results also provide a comprehensive way for validating the accuracy of the algorithm proposed in Section III-C for detecting fractionations in the QRS-complex. Ignoring the cases of confounder misclassification due to the axis mismatch and the less accurate QRS-onset localization, it is evident that only in one case (record 36) the proposed fractionation detection algorithm fails to detect a notch, thereby giving a fractionation detection accuracy of approximately $98 \%$.

TABLE I

CATEGORISATION DISCREPANCIES

\begin{tabular}{|c|c|c|c|c|}
\hline System variant & \# Rec & Cardiologists & Automated System & Explanation \\
\hline \multirow{3}{*}{ SASS } & 27 & LAFB & No Conf. & Axis value \\
& 36 & LAFB & LBBB & Undetected notch \\
& 44 & No Conf. & LAFB & Axis value \\
\hline \multirow{2}{*}{ add FASS } & 11 & LBBB & No Conf. & Ignored notch \\
& 48 & LAFB & LBBB & Axis value \\
\hline
\end{tabular}

TABLE II

CARdiologists and Automated System AXIS VAlues For RECORdS 27,44 AND 48

\begin{tabular}{|c|c|c|}
\hline \# Rec & Cardiologists & Automated System \\
\hline 27 & $\left(-90^{\circ}\right)$ & $\left(-23^{\circ}\right.$-both $)$ \\
44 & $\left(-30^{\circ}\right)$ & $\left(-45.4^{\circ}\right.$-SASS $),\left(-57^{\circ}\right.$-FASS $)$ \\
48 & $\left(-30^{\circ}\right)$ & $\left(-47^{\circ}\right.$-FASS $)$ \\
\hline
\end{tabular}

2) Validation of the Selvester Scoring: The comparisons of the manually calculated Selvester score to both the SASS and FASS systems are shown in Fig. 11 in the form of histograms of the absolute difference in the scores of the 51 patients. For those records where confounders were found to be different, the final score calculation in the automated implementation (both SASS and FASS) was performed using the confounding categories of the cardiologists. It can be seen that the SASS 
system is able to match the manual scoring exactly in 45 out of 51 records whereas for another 3 records it calculate the score within 1 point of the manual score. It is to be noted that in the clinical practice the difference of 1 point is not considered significant as it is well within the limit of human error. As a consequence it could be concluded that the proposed algorithm in its stand-alone form (i.e. SASS) exhibits an approximate accuracy of $94 \%$ in computing the final score. On the other hand the FASS system exhibits complete agreement with the manual scoring in 42 out of 51 records and an additional 5 records are within 1-point difference, therefore attaining an accuracy of approximately $92 \%$. It is worth pointing out at this stage that, while digitizing the paper ECGs, we have observed some loss of resolution, which quite understandably may have affected the final score values. However, despite this fact, the accuracy exhibited by the proposed system makes it quite promising.

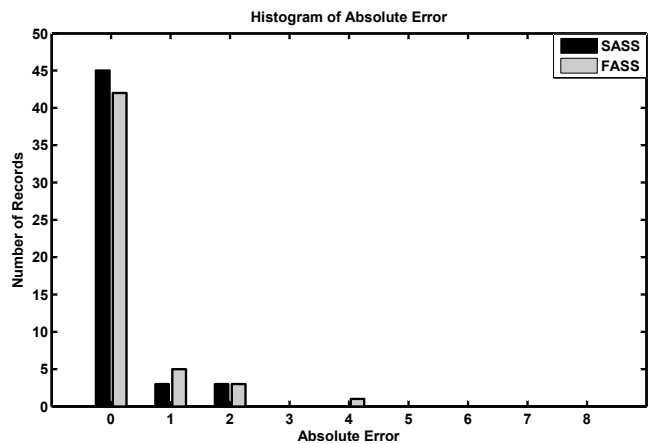

Fig. 11. Histogram of the absolute difference between the Cardiologists and the Automated System's derived Selvester score

As mentioned earlier, while evaluating the performance of the FASS system, we have used 25 records with the same heartbeat that was used for the SASS system (manually annotated by the cardiologists) in order to examine the dependence of the FASS system on the TDMG algorithm used for extracting the necessary ECG parameters (P-onset/offset, QRS-onset/offset). Fig.12 shows that under this condition 23 out of 25 records are accurately scored by the SASS system whereas the same level of accuracy is observed in 20 out of 25 records for the FASS system. The two records that are scored differently than the cardiologists in SASS, are also scored differently in FASS. Surprisingly, the scores produced by SASS and FASS in these are the same. In the additional three records scored differently only by FASS, two records are scored within 1-point difference and one record is scored within 2-points difference. From the above results it is evident that not only the proposed algorithm in its stand-alone form gives high level of accuracy for calculating the Selvester score, but also a fully automated system integrating the proposed algorithm with the TDMG (resulting in the FASS system) shows comparable level of accuracy.

\section{CONCLUSIONS}

In this paper we have proposed a novel system for the automated computation of the updated Selvester score that may enable quantifying myocardial scar from less expensive

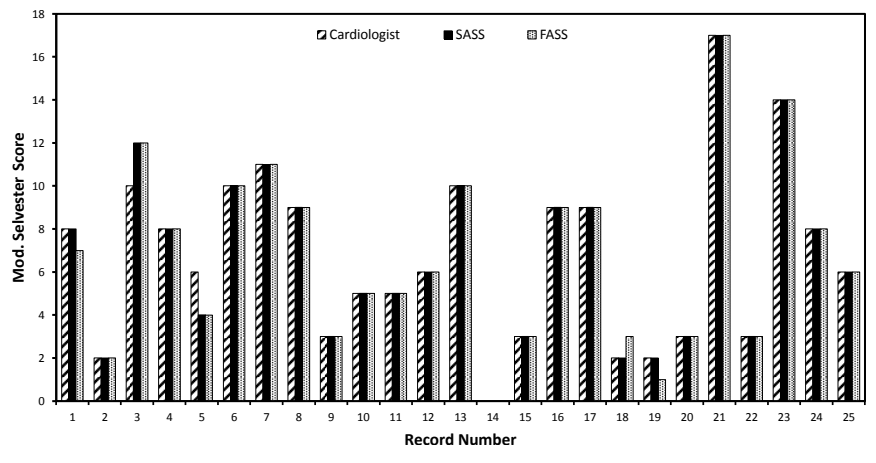

Fig. 12. Comparison of the FASS and SASS systems against the Cardiologists score on 25 records where the same beat was used for calculating the Selvester score

ECG recordings in the primary care. The novel algorithm based on SWT for detecting and classifying fractionated QRS proposed here, as the core of the system, exhibits approximately $98 \%$ accuracy. Our validation results show that the proposed automated Selvester scoring system achieves $94 \%$ accuracy both in classifying the confounders as well as in computing the actual Selvester score which is well acceptable in the clinical community. Such high level of accuracy makes the proposed system a potential candidate for a fast and large-volume screening tool for scar estimation in the primary care from low-cost ECG recordings resulting in significant economic benefits in care delivery.

\section{REFERENCES}

[1] R. H. Selvester, J. C. Solomon, and T. L. Gillespie, "Digital Computer Model of a Total Body Electrocardiographic Surface Map: An Adult Male-Torso Simulation with Lungs," Circ., vol. 38, pp. 684-690, 1968.

[2] R. E. Ideker, G. S. Wagner, W. K. Ruth, D. R. Alonso, S. P. Bishop, C. M. Bloor, J. T. Fallon, G. J. Gottlieb, D. B.Hackel, H. R. Phillips, K. A. Reimer, S. F. Roark, W. J. Rogers, R. M. Savage, R. D. White, R. H. Selvester, "Evaluation of a QRS scoring system for estimating myocardial infarct size. II. Correlation with quantitative anatomic findings for anterior infarcts." Am J Cardiol, vol. 49(7), pp. 1604-1614, 1982.

[3] G. S. Wagner, C. J.Freye, S. T. Palmeri, S. F. Roark, N. C. Stack, R. E. Ideker, F. E. Harrell Jr, R. H. Selvester, "Evaluation of a QRS scoring system for estimating myocardial infarct size. I. Specificity and observer agreement." Circulation, vol. 65(2), pp. 342-347, 1982.

[4] D. G. Strauss, R. H. Selvester, J. A. Lima, H. Arheden, J. M. Miller, G. Gerstenblith, E. Marbn, R. G. Weiss, G. F. Tomaselli, G. S. Wagner, K. C. Wu, "ECG Quantification of Myocardial Scar in Cardiomyopathy Patients with or without Conduction Defects: Correlation with Cardiac Magnetic Resonance and Arrhythmogenesis," Circ Arrhythm Electrophysiol, vol. 1(5), pp. 327-336, 2008.

[5] D. G. Strauss and R. H. Selvester, "The QRS complexa biomarker that images the heart: QRS scores to quantify myocardial scar in the presence of normal and abnormal ventricular conduction," J. Electrocardiol, vol. 42 , no. 1 , pp. $85-96,2009$.

[6] Z. Loring, S. Chelliah, R. H. Selvester, G. S. Wagner, D. G. Strauss “A detailed guide for quantification of myocardial scar with the Selvester QRS score in the presence of electrocardiogram confounders," J. Electrocardiol, vol. 44, no. 5, pp. 544 - 554, 2011.

[7] B. M. Horáček, J. W. Warren, A. Albano, M. A. Palmeri, J. C. Rembert, J. C. Greenfield Jr, G. S. Wagner. "Development of an automated Selvester Scoring System for estimating the size of myocardial infarction from the electrocardiogram," J. Electrocardiol., vol. 39(2), pp. 162 168, 2006.

[8] J. E. Pope, N. B Wagner, D. Dubow, J. H. Edmonds, G. S. Wagner, W. K. Haisty Jr, "Development and validation of an automated method of the selvester QRS scoring system for myocardial infarct size," Am J Cardiol, vol. 61, no. 10, pp. $734-738,1988$ 
[9] W. K. Haisty Jr, O. Pahlm, N. B. Wagner, J. E. Pope, G. S. Wagner, "Performance of the automated complete Selvester QRS scoring system in normal subjects and patients with single and multiple myocardial infarctions," J. Am Col. Card., vol. 19(2), pp. 341 - 346, 1992.

[10] B.-U. Kohler, C. Hennig, and R. Orglmeister, "The principles of software QRS detection," IEEE Eng. Med. Biol. Mag., vol. 21, no. 1, pp. 42 -57, Jan.-Feb. 2002.

[11] F. Badilini, T. Erdem, W. Zareba, A. J. Moss, "ECGScan: a method for conversion of paper electrocardiographic printouts to digital electrocardiographic files." J Electrocardiol, vol. 38, no. 4, pp. 310-8, 2005.

[12] P. de Chazal, M. O'Dwyer, and R. Reilly, "Automatic classification of heartbeats using ECG morphology and heartbeat interval features," IEEE Trans. Biomed. Eng., vol. 51, no. 7, pp. 1196 -1206, Jul 2004.

[13] J. Martinez, R. Almeida, S. Olmos, A. P. Rocha, P. Laguna, "A waveletbased ECG delineator: evaluation on standard databases," IEEE Trans. Biomed. Eng., vol. 51, no. 4, pp. 570 -581, Apr 2004.

[14] C. Li, C. Zheng, and C. Tai, "Detection of ECG characteristic points using wavelet transforms," IEEE Trans. Biomed. Eng., vol. 42, no. 1, pp. $21-28$, Jan 1995.

[15] J. Sahambi, S. Tandon, and R. Bhatt, "Using wavelet transforms for ECG characterization. An on-line digital signal processing system," IEEE Eng Med. Biol Mag, vol. 16, no. 1, pp. 77 -83, Jan 1997.

[16] Z. R. Struzik and A. Siebes, "The haar wavelet transform in the time series similarity paradigm," in $P K D D, 1999$, pp. 12-22.

[17] S. Mallat, "Zero-crossings of a wavelet transform," IEEE Trans. Inf. Theory, vol. 37, no. 4, pp. 1019-1033, Jul 1991.

[18] M. Shensa, "The discrete wavelet transform: wedding the à trous and Mallat algorithms," IEEE Trans. Sig. Process., vol. 40, no. 10, pp. 2464 2482, Oct, 1992.

[19] H. Burger and J. van Milaan, "Heart-vector and leads. Part III: geometrical representation," Br Heart J., vol. 10, pp. 229-233, Oct, 1948.

[20] F. R. Baltazar, Basic and Bedside Electrocardiography. Baltimore MD, USA: Lippincott Williams \& Wilkins, 2009.

[21] E. B Mazomenos, T. Chen, A. Acharyya, A. Bhattacharya, J. Rosengarten, K. Maharatna, "A time-domain morphology and gradient based algorithm for ECG feature extraction," in IEEE ICIT, Mar, 2012.

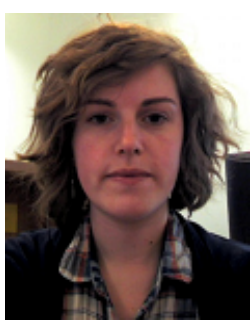

Valentina Bono received the M.Sc. degree in Biomedical Engineering from the University of Pisa, Pisa, IT, in 2012. She is currently working towards her Ph.D. degree in biomedical engineering in the Electronics and Software Systems Group in the University of Southampton.

Her research interests include biomedical signal processing and image processing.

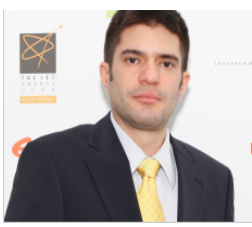

Evangelos B. Mazomenos received a Diploma degree (5-year) in Electrical \& Computer Engineering from the Univ. of Patras, Greece and a PhD from the School of ECS, Univ. of Southampton, UK in 2006 and 2012 respectively. He is the recipient of the 2009 IET Leslie H. Paddle fellowship on postgraduate studies for his $\mathrm{PhD}$ research on real-time target tracking in WSN. From January 2011 he has been a research fellow in ECS.

His research interests are in the area of WSNs with a focus on positioning and tracking, biomedical signal processing and dynamic estimation algorithms.

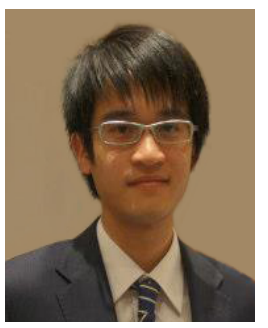

Taihai Chen received the B.Eng. degree (1st Class Hons.) from University of Electronic Science and Technology of China (UESTC), Chengdu, China in 2009, and later received the M.Sc. degree (Distinction) from University of Southampton, Southampton, UK in 2010. Since October 2010, he has been working towards his Ph.D degree in Electronic and Software Systems (ESS) research group in the University of Southampton.

His research interests include biomedical signal processing, low-power design techniques, pattern recognition, machine learning, associated VLSI architectures, and nextgeneration healthcare systems.

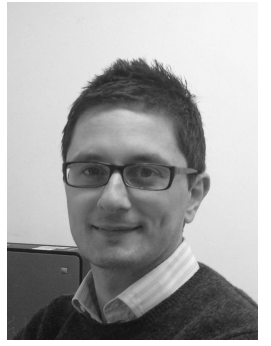

James Rosengarten received his MBBS from the University of London in 2002 and became a member of of the Royal College of Physicians (MRCP(UK)) in 2005. Currently he is a clinical cardiologist based in Southampton. Following training in general cardiology in London and Wessex, he is now specialising in heart rhythm disorders, including invasive testing and implantable devices. He is currently the Research Fellow in Cardiac Rhythm Management, based at University Hospital Southampton. Under the supervision of Prof. Morgan and Prof. Hanson at the University of Southampton, he is utilising engineering techniques to discover novel biomarkers of sudden cardiac death risk.

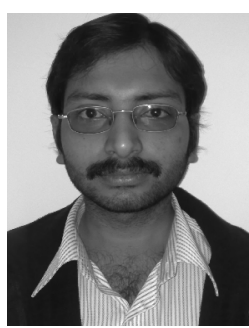

Amit Acharyya (M13) received the Ph.D. degree in 2011 from the School of Electronics and Computer Science in the University of Southampton, UK. Currently he is working as an Assistant Professor in the Indian Institute of Technology (IIT), Hyderabad, India.

His research interests include signal processing algorithms, VLSI architectures, low power design techniques, computer arithmetic, numerical analysis, linear algebra, bio-informatics and electronic aspects of pervasive computing.

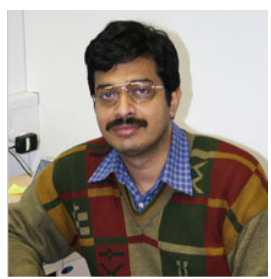

Koushik Maharatna (M02) received the M.Sc. degree in electronic science from Calcutta University, Calcutta, India, in 1995 and the Ph.D. degree from Jadavpur University, Calcutta, India, in 2002. From 1996 to 2000, he was involved in different projects sponsored by the Government of India undertaken at the Indian Institute of Technology (IIT), Kharagpur, India. From 2000 to 2003, he was a Research Scientist in IHP, Frankfurt (Oder), Germany. During this phase, his main involvement was related to the design of a single-chip modem for the IEEE 802.11a standard. In September 2006, he joined the School of Electronics and Computer Science of the University of Southampton, U.K., where he is currently a Reader. His research interests include low-power VLSI and signal processing for applications in DSP, communication and next-generation healthcare systems, computer arithmetic, analog signal processing, and bioinspired circuits and systems.

Dr. Maharatna is a member of the IEEE VLSI System Application (VSA) Technical Committee.

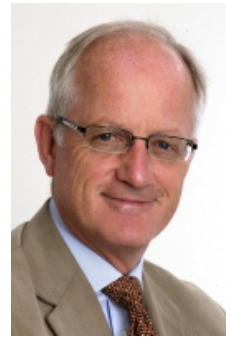

John Morgan graduated from the University of Cambridge with a BA in Natural Sciences in 1979 and from the Westminster Medical School with an MB BChir in 1982. He then obtained an MA and an MD from Cambridge University in 1983 and 1991 respectively. He became a member of the Royal College of Physicians (MRCP(UK)) in 1985 and Fellow (FRCP) in 1995. On his appointment as Consultant Cardiologist, Wessex Cardiothoracic Centre (1992), he founded the Wessex Cardiac Arrhythmia Management Service which has become recognised as a centre of clinical and research excellence. He was appointed an honorary professor at the University of Teesside in 2006 for his work in medical education and received a personal chair in the School of Medicine, University of Southampton in 2007. Professor Morgan holds several published patents relating to invention of novel interventional and device technologies.

$\mathrm{He}$ supervises/has supervised several fellows in doctoral research projects and continues to research and publish extensively and is an internationally recognised leader in his field. He also sits on a series of national and international boards/professional associations and governing bodies. 


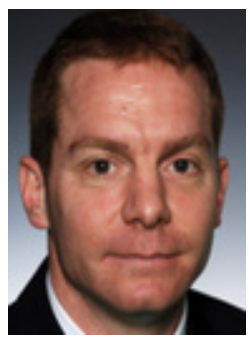

Nick Curzen is a Consultant Cardiologist at University Southampton Hospitals and Professor of Interventional Cardiology at the University of Southampton. Betwenn 1993-1996 he trained in cardiology at Southampton, Bournemouth, Royal Brompton, London Chest and St Bartholomews hospitals and completed a $\mathrm{PhD}$ in vascular biology at Imperial College, London. He has wide ranging research interests including platelet function, myocardial ischaemia, cardiac magnetic resonance and scar. He has over 130 peer review scientific papers and has edited 3 cardiology textbooks.

In November 2010, Nick Curzen came top of a national poll of interventional cardiologist published in the Daily Mail. 